International Mathematical Forum, Vol. 9, 2014, no. 29, 1389 - 1396

HIKARI Ltd, www.m-hikari.com

http://dx.doi.org/10.12988/imf.2014.47141

\title{
Remarks on Fuglede-Putnam Theorem for Normal Operators Modulo the Hilbert-Schmidt Class
}

\author{
Vasile Lauric \\ Department Mathematics \\ Florida A\&M University \\ Tallahassee, FL 32307 USA
}

Copyright (c) 2014 Vasile Lauric. This is an open access article distributed under the Creative Commons Attribution License, which permits unrestricted use, distribution, and reproduction in any medium, provided the original work is properly cited.

\begin{abstract}
In a recent note, we proved a Fuglede-Putnam commutativity theorem for almost normal operators with finite modulus of $\mathcal{C}_{2}$-quasitriangularity modulo the HilbertSchmidt class. In this note we show how our proof can be adjusted to the case of normal operators to obtain an optimal norm estimate obtained by G. Weiss. The result is also reviewed in the case of almost normal operators with zero modulus of $\mathcal{C}_{2}$-quasitriangularity for the operator and its adjoint and an example is provided.
\end{abstract}

Mathematics Subject Classification: 47B20

Keywords: Hilbert-Schmidt and trace-class operators, $\mathcal{C}_{2}$-quasitriangularity modulus

Let $\mathcal{H}$ be a separable, infinite dimensional, complex Hilbert space, and denote by $L(\mathcal{H})$ the algebra of all bounded linear operators on $\mathcal{H}$ and by $\mathcal{C}_{1}(\mathcal{H})$ and $\mathcal{C}_{2}(\mathcal{H})$ the trace class and Hilbert-Schmidt class, respectively. For an operator $T \in L(\mathcal{H})$, let $D_{T}$ denote its self-commutator, that is $T^{*} T-T T^{*}$. An operator $T \in L(\mathcal{H})$ for which $D_{T} \in \mathcal{C}_{1}$ is called almost normal. We will denote the class of almost normal operators on $\mathcal{H}$ by $A N(\mathcal{H})$.

The classical Fuglede-Putnam theorem states if $M, N \in L(\mathcal{H})$ are normal operators and $X \in L(\mathcal{H})$ is such that $M X=X N$, then $M^{*} X=X N^{*}$. G. 
Weiss (see $[8,9]$ ) extended this result by proving that if $M, N \in L(\mathcal{H})$ are normal operators and $X \in L(\mathcal{H})$ such that $M X-X N \in \mathcal{C}_{2}(\mathcal{H})$, then $M^{*} X-$ $X N^{*} \in \mathcal{C}_{2}(\mathcal{H})$ and

$$
\left\|M^{*} X-X N^{*}\right\|_{2}=\|M X-X N\|_{2} .
$$

T. Furuta ([1]) and F. Kittaneh ([2]) extended the above result to subnormal operators (a subnormal operator is the restriction of a normal operator to an invariant subspace) by proving that if $M, N^{*} \in L(\mathcal{H})$ are subnormal operators and $X \in L(\mathcal{H})$ such that $M X-X N \in \mathcal{C}_{2}(\mathcal{H})$, then $M^{*} X-X N^{*} \in \mathcal{C}_{2}(\mathcal{H})$ and

$$
\left\|M^{*} X-X N^{*}\right\|_{2} \leq\|M X-X N\|_{2} .
$$

Kittaneh [2] provided some result that suggested that it would be interesting to investigate Fuglede-Putnam generalizations in which operators $M, N$ are almost normal. For instance, he proved that if $S \in L(\mathcal{H})$ is an almost normal subnormal operator and $S X-X S \in \mathcal{C}_{2}(\mathcal{H})$ for some $X \in L(\mathcal{H})$, then $S^{*} X-X S^{*} \in \mathcal{C}_{2}(\mathcal{H})$.

In [3] we extended Fuglede-Putnam theorem to operators that are almost normal operators that have finite modulus of $\mathcal{C}_{2}$-quasitriangularity modulo Hilbert-Schmidt class.

Let $\mathcal{P}(\mathcal{H})$, (or simply $\mathcal{P}$ ) denote the set of all finite rank orthogonal projections on $\mathcal{H}$. Recall (see [4]) that the modulus of $\mathcal{C}_{2}$-quasitriangularity of an operator $T \in L(\mathcal{H})$ is

$$
q_{2}(T)=\liminf _{P \in \mathcal{P}}\|(I-P) T P\|_{2},
$$

where liminf is with respect to the natural order on $\mathcal{P}$.

Recall that if $m(T)$ denotes the rational cyclicity of $T$, then ([6])

$$
q_{2}(T) \leq(m(T))^{\frac{1}{2}}\|T\|,
$$

that is, operators of finite rational cyclicity have finite modulus of $\mathcal{C}_{2}$-quasitriangularity.

According to [7], if $T \in A N(\mathcal{H})$ and $q_{2}(T)<\infty$, then $q_{2}\left(T^{*}\right)<\infty$ and there exists a sequence $P_{n} \in \mathcal{P}, n \geq 1$, with $P_{n} \uparrow I$ strongly, so that

$$
\lim _{n \rightarrow \infty}\left\|\left(I-P_{n}\right) T P_{n}\right\|_{2}=q_{2}(T) \text { and } \lim _{n \rightarrow \infty}\left\|\left(I-P_{n}\right) T^{*} P_{n}\right\|_{2}=q_{2}\left(T^{*}\right),
$$

and

$$
q_{2}^{2}(T)=q_{2}^{2}\left(T^{*}\right)+\operatorname{Tr}\left(D_{T}\right) .
$$

We will use the following idea that was first used by T. Furuta [1], namely if $T_{1}, T_{2} \in L(\mathcal{H})$, then the operator $\Delta_{T_{1}, T_{2}}(X)=T_{1} X-X T_{2}$ defined on the Hilbert space $\mathcal{C}_{2}(\mathcal{H})$ (with inner product $\langle X, Y\rangle_{2}=\operatorname{Tr}\left(X Y^{*}\right)$ ) has its adjoint 
given by $\Delta_{T_{1}, T_{2}}^{*}(X)=T_{1}^{*} X-X T_{2}^{*}$ and thus the self-commutator of $\Delta_{12}:=$ $\Delta_{T_{1}, T_{2}}$ is

$$
D_{\Delta_{12}}(X)=D_{T_{1}} X-X D_{T_{2}}=\Delta_{D_{T_{1}}, D_{T_{2}}}(X) .
$$

Using operator theoretic concepts, we proved in [3] the following.

Theorem 1. Let $S \in A N(\mathcal{H})$ with $q_{2}(S)<\infty$ and let $X \in L(\mathcal{H})$ such that $R:=S X-X S \in \mathcal{C}_{2}(\mathcal{H})$. Then $Q:=S^{*} X-X S^{*} \in \mathcal{C}_{2}(\mathcal{H})$.

The proof of Theorem 1 provided the estimate

$$
\|Q\|_{2}^{2} \leq 4\|R\|_{2}^{2}+\|X\|^{2}\left(8\left\|D_{S}\right\|_{1}+30 q_{2}^{2}(S)+26 q_{2}^{2}\left(S^{*}\right)\right)
$$

which implies that for a normal operator $S \in L(\mathcal{H}),\|Q\|_{2} \leq 2\|R\|_{2}$, (since for a normal operator $S, q_{2}(S)=q_{2}\left(S^{*}\right)=\left\|D_{S}\right\|_{1}=0$.)

The purpose of this note is to review the proof given in [3] and show that in the case of a normal operator $S \in L(\mathcal{H})$, it gives the optimal estimate, namely

$$
\left\|S^{*} X-X S^{*}\right\|_{2}=\|S X-X S\|_{2} .
$$

The proof depends only operator theoretic concepts rather than using generating functions defined by $\mathrm{G}$. Weiss.

Proposition 2. Let $S \in L(\mathcal{H})$ be a normal operator and let $X \in L(\mathcal{H})$ such that $R:=S X-X S \in \mathcal{C}_{2}(\mathcal{H})$. Then $Q:=S^{*} X-X S^{*} \in \mathcal{C}_{2}(\mathcal{H})$ and $\|Q\|_{2} \leq\|R\|_{2}$.

Proof. Let $P_{n} \in \mathcal{P}, n \geq 1$, such that $P_{n} \uparrow I$ and

$$
\lim _{n \rightarrow \infty}\left\|\left(I-P_{n}\right) S P_{n}\right\|_{2}=q_{2}(S) \text { and } \lim _{n \rightarrow \infty}\left\|\left(I-P_{n}\right) S^{*} P_{n}\right\|_{2}=q_{2}\left(S^{*}\right)<\infty .
$$

It is known now $([5])$ that for a normal operator $S, q_{2}(S)=q_{2}\left(S^{*}\right)=0$.

Then $\left\|Q P_{n}\right\|_{2}^{2}=\operatorname{Tr}\left(P_{n} Q^{*} Q P_{n}\right) \uparrow \operatorname{Tr}\left(Q^{*} Q\right)$. We need to prove that the sequence $\left\{\left\|Q P_{n}\right\|_{2}\right\}_{n}$ is bounded above. Write

$$
S=\left(\begin{array}{ll}
S_{1 n} & S_{2 n} \\
S_{3 n} & S_{4 n}
\end{array}\right) \text { and } X=\left(\begin{array}{cc}
X_{1 n} & X_{2 n} \\
X_{3 n} & X_{4 n}
\end{array}\right)
$$

relative to the decomposition of $\mathcal{H}$ as $P_{n} \mathcal{H} \oplus\left(I-P_{n}\right) \mathcal{H}$. Thus

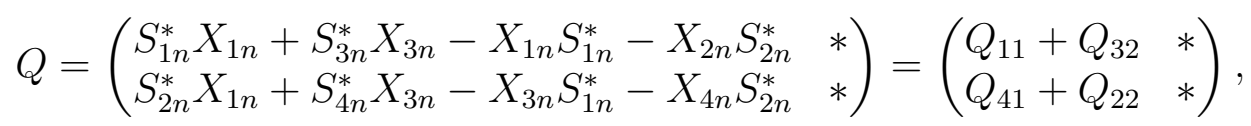

where $Q_{11}=S_{1 n}^{*} X_{1 n}-X_{1 n} S_{1 n}^{*}, \quad Q_{32}=S_{3 n}^{*} X_{3 n}-X_{2 n} S_{2 n}^{*}, \quad Q_{41}=S_{4 n}^{*} X_{3 n}-$ $X_{3 n} S_{1 n}^{*}$, and $Q_{22}=S_{2 n}^{*} X_{1 n}-X_{4 n} S_{2 n}^{*}$. Consequently, $\left\|Q P_{n}\right\|_{2}^{2}=\| Q_{11}+$ $Q_{32}\left\|_{2}^{2}+\right\| Q_{41}+Q 22 \|_{2}^{2}$. 
Next we estimate the Hilbert-Schmidt norm of each of the $Q$ 's. We begin with $Q_{32}$, namely

$$
\begin{aligned}
\left\|Q_{32}\right\|_{2}^{2} & =\left\|S_{3 n}^{*} X_{3 n}-X_{2 n} S_{2 n}^{*}\right\|_{2}^{2} \leq\left(\|X\|\left\|S_{3 n}^{*}\right\|_{2}+\|X\|\left\|S_{2 n}^{*}\right\|_{2}\right)^{2} \leq \\
& \leq 2\|X\|^{2}\left(\left\|S_{3 n}^{*}\right\|_{2}^{2}+\left\|S_{2 n}^{*}\right\|_{2}^{2}\right) \rightarrow 2\|X\|^{2}\left(q_{2}^{2}(S)+q_{2}^{2}\left(S^{*}\right)\right),
\end{aligned}
$$

and thus

$$
\left\|Q_{32}\right\|_{2} \rightarrow 0
$$

(recall that all $Q^{\prime}$ s depend on $n$.)

Denote $\Delta_{11}(X)=S_{1 n} X-X S_{1 n}$ defined on $\mathcal{C}_{2}\left(P_{n} \mathcal{H}\right)$; therefore

$$
Q_{11}=\Delta_{11}^{*}\left(X_{1 n}\right)=S_{1 n}^{*} X_{1 n}-X_{1 n} S_{1 n}^{*},
$$

and thus

$$
\left[\Delta_{11}^{*}, \Delta_{11}\right]\left(X_{1 n}\right)=D_{S_{1 n}} X_{1 n}-X_{1 n} D_{S_{1 n}}
$$

and then

$$
\begin{gathered}
\left\|\Delta_{11}\left(X_{1 n}\right)\right\|_{2}^{2}-\left\|\Delta_{11}^{*}\left(X_{1 n}\right)\right\|_{2}^{2}=\left\langle\left[\Delta_{11}^{*}, \Delta_{11}\right]\left(X_{1 n}\right), X_{1 n}\right\rangle_{2}= \\
=\operatorname{Tr}\left(D_{S_{1 n}} X_{1 n} X_{1 n}^{*}\right)-\operatorname{Tr}\left(X_{1 n} D_{S_{1 n}} X_{1 n}^{*}\right)= \\
=\operatorname{Tr}\left(X_{1 n}^{*} D_{S_{1 n}} X_{1 n}\right)-\operatorname{Tr}\left(X_{1 n} D_{S_{1 n}} X_{1 n}^{*}\right) .
\end{gathered}
$$

A matrix calculation shows that $P_{n} D_{S} P_{n}=D_{S_{1 n}}+S_{3 n}^{*} S_{3 n}-S_{2 n} S_{2 n}^{*}$, and thus

$$
X_{1 n}^{*} D_{S_{1 n}} X_{1 n}=X_{1 n}^{*} D_{S} X_{1 n}-X_{1 n}^{*} S_{3 n}^{*} S_{3 n} X_{1 n}+X_{1 n}^{*} S_{2 n} S_{2 n}^{*} X_{1 n}
$$

and

$$
X_{1 n} D_{S_{1 n}} X_{1 n}^{*}=X_{1 n} D_{S} X_{1 n}^{*}-X_{1 n} S_{3 n}^{*} S_{3 n} X_{1 n}^{*}+X_{1 n} S_{2 n} S_{2 n}^{*} X_{1 n}^{*},
$$

respectively. Therefore

$$
\begin{gathered}
\left\|\Delta_{11}^{*}\left(X_{1 n}\right)\right\|_{2}^{2}-\left\|\Delta_{11}\left(X_{1 n}\right)\right\|_{2}^{2}=-\operatorname{Tr}\left(X_{1 n}^{*} D_{S_{1 n}} X_{1 n}\right)+\operatorname{Tr}\left(X_{1 n} D_{S_{1 n}} X_{1 n}^{*}\right)= \\
\operatorname{Tr}\left(X_{1 n} D_{S} X_{1 n}^{*}\right)-\operatorname{Tr}\left(X_{1 n} S_{3 n}^{*} S_{3 n} X_{1 n}^{*}\right)+\operatorname{Tr}\left(X_{1 n} S_{2 n} S_{2 n}^{*} X_{1 n}^{*}\right)- \\
-\operatorname{Tr}\left(X_{1 n}^{*} D_{S} X_{1 n}\right)+\operatorname{Tr}\left(X_{1 n}^{*} S_{3 n}^{*} S_{3 n} X_{1 n}\right)-\operatorname{Tr}\left(X_{1 n}^{*} S_{2 n} S_{2 n}^{*} X_{1 n}\right) \leq \\
\leq \operatorname{Tr}\left(X_{1 n} D_{S} X_{1 n}^{*}\right)-\operatorname{Tr}\left(X_{1 n}^{*} D_{S} X_{1 n}\right)+ \\
+\operatorname{Tr}\left(X_{1 n} S_{2 n} S_{2 n}^{*} X_{1 n}^{*}\right)+\operatorname{Tr}\left(X_{1 n}^{*} S_{3 n}^{*} S_{3 n} X_{1 n}\right) \leq \\
\leq 2\|X\|^{2}\left\|D_{S}\right\|_{1}+\left\|S_{2 n}^{*} X_{1 n}^{*}\right\|_{2}^{2}+\left\|S_{3 n} X_{1 n}\right\|_{2}^{2} \rightarrow \\
\rightarrow\|X\|^{2}\left(2\left\|D_{S}\right\|_{1}+q_{2}^{2}(S)+q_{2}^{2}\left(S^{*}\right)\right),
\end{gathered}
$$

and consequently

$$
\left\|Q_{11}\right\|_{2} \lesssim\left\|R_{11}\right\|_{2}
$$

where $R_{11}=S_{1 n} X_{1 n}-X_{1 n} S_{1 n}=\Delta_{11}\left(X_{1 n}\right)$, and $\lesssim$ means that the difference between right hand side and left hand side is bounded above by a non-negative sequence that converges to 0 . Combining (1) and (2), one obtains

$$
\left\|Q_{11}+Q_{32}\right\|_{2} \leq\left\|Q_{11}\right\|_{2}+\left\|Q_{32}\right\|_{2} \lesssim\left\|R_{11}\right\|_{2}
$$


The operator $Q_{41}=S_{4 n}^{*} X_{3 n}-X_{3 n} S_{1 n}^{*}$ can be viewed as $\Delta_{41}^{*}\left(X_{3 n}\right)$, where $\Delta_{41}^{*}$ is the adjoint of

$$
\Delta_{41}: \mathcal{C}_{2}\left(P_{n} \mathcal{H},\left(I-P_{n}\right) \mathcal{H}\right) \rightarrow \mathcal{C}_{2}\left(P_{n} \mathcal{H},\left(I-P_{n}\right) \mathcal{H}\right)
$$

defined by $\Delta_{41}(Y)=S_{4 n} Y-Y S_{1 n}$. Denoting $\Delta_{41}\left(X_{3 n}\right)$ by $R_{41}$, we have

$$
\begin{gathered}
\left\|R_{41}\right\|_{2}^{2}-\left\|Q_{41}\right\|_{2}^{2}=\left\|\Delta_{41}\left(X_{3 n}\right)\right\|_{2}^{2}-\left\|\Delta_{41}^{*}\left(X_{3 n}\right)\right\|_{2}^{2}= \\
=\left\langle\left[\Delta_{41}^{*}, \Delta_{41}\right]\left(X_{3 n}\right), X_{3 n}\right\rangle_{2}=\operatorname{Tr}\left(D_{S_{4 n}} X_{3 n} X_{3 n}^{*}\right)-\operatorname{Tr}\left(X_{3 n} D_{S_{1 n}} X_{3 n}^{*}\right)= \\
=\operatorname{Tr}\left(X_{3 n}^{*} D_{S_{4 n}} X_{3 n}\right)-\operatorname{Tr}\left(X_{3 n} D_{S_{1 n}} X_{3 n}^{*}\right) .
\end{gathered}
$$

Since $X_{3 n}=\left(I-P_{n}\right) X P_{n}$, and thus $X_{3 n}^{*}=P_{n} X^{*}\left(I-P_{n}\right)$, and using again the matrix representation of $D_{S}$ relative to the decomposition of $\mathcal{H}$ into $P_{n} \mathcal{H} \oplus$ $\left(I-P_{n}\right) \mathcal{H}$, that is $P_{n} D_{S} P_{n}=D_{S_{1 n}}+S_{3 n}^{*} S_{3 n}-S_{2 n} S_{2 n}^{*}$, we obtain

$$
\begin{aligned}
& X_{3 n} D_{S_{1 n}} X_{3 n}^{*}=X_{3 n}\left(D_{S}-S_{3 n}^{*} S_{3 n}+S_{2 n} S_{2 n}^{*}\right) X_{3 n}^{*}= \\
& =X_{3 n} D_{S} X_{3 n}^{*}-X_{3 n} S_{3 n}^{*} S_{3 n} X_{3 n}^{*}+X_{3 n} S_{2 n} S_{2 n}^{*} X_{3 n}^{*}
\end{aligned}
$$

On other hand $\left(I-P_{n}\right) D_{S}\left(I-P_{n}\right)=D_{S_{4 n}}+S_{2 n}^{*} S_{2 n}-S_{3 n} S_{3 n}^{*}$, which implies

$$
\begin{aligned}
& X_{3 n}^{*} D_{S_{4 n}} X_{3 n}=X_{3 n}^{*}\left(D_{S}-S_{2 n}^{*} S_{2 n}+S_{3 n} S_{3 n}^{*}\right) X_{3 n}= \\
& =X_{3 n}^{*} D_{S} X_{3 n}-X_{3 n}^{*} S_{2 n}^{*} S_{2 n} X_{3 n}+X_{3 n}^{*} S_{3 n} S_{3 n}^{*} X_{3 n}
\end{aligned}
$$

Thus

$$
\begin{gathered}
\left\|Q_{41}\right\|_{2}^{2}-\left\|R_{41}\right\|_{2}^{2}=-\operatorname{Tr}\left(X_{3 n}^{*} D_{S_{4 n}} X_{3 n}\right)+\operatorname{Tr}\left(X_{3 n} D_{S_{1 n}} X_{3 n}^{*}\right)= \\
=\operatorname{Tr}\left(X_{3 n} D_{S} X_{3 n}^{*}\right)-\operatorname{Tr}\left(X_{3 n} S_{3 n}^{*} S_{3 n} X_{3 n}^{*}\right)+\operatorname{Tr}\left(X_{3 n} S_{2 n} S_{2 n}^{*} X_{3 n}^{*}\right)+ \\
-\operatorname{Tr}\left(X_{3 n}^{*} D_{S} X_{3 n}\right)+\operatorname{Tr}\left(X_{3 n}^{*} S_{2 n}^{*} S_{2 n} X_{3 n}\right)-\operatorname{Tr}\left(X_{3 n}^{*} S_{3 n} S_{3 n}^{*} X_{3 n}\right) \leq \\
\leq \operatorname{Tr}\left(X_{3 n} D_{S} X_{3 n}^{*}\right)-\operatorname{Tr}\left(X_{3 n}^{*} D_{S} X_{3 n}\right)+ \\
+\operatorname{Tr}\left(X_{3 n} S_{2 n} S_{2 n}^{*} X_{3 n}^{*}\right)+\operatorname{Tr}\left(X_{3 n}^{*} S_{2 n}^{*} S_{2 n} X_{3 n}\right) \leq \\
\leq 2\|X\|^{2}\left\|D_{S}\right\|_{1}+\left\|S_{2 n}^{*} X_{3 n}^{*}\right\|_{2}^{2}+\left\|S_{2 n} X_{3 n}\right\|_{2}^{2} \rightarrow \\
\rightarrow\|X\|^{2}\left(2\left\|D_{S}\right\|_{1}+2 q_{2}^{2}\left(S^{*}\right)\right),
\end{gathered}
$$

so

$$
\left\|Q_{41}\right\|_{2} \lesssim\left\|R_{41}\right\|_{2}
$$

Finally, $Q_{22}=S_{2 n}^{*} X_{1 n}-X_{4 n} S_{2 n}^{*}$ can be handled similarly to $Q_{32}$, namely

$$
\left\|Q_{22}\right\|_{2} \leq\left\|S_{2 n}^{*}\right\|_{2}\left\|X_{1 n}\right\|+\left\|X_{4 n}\right\|\left\|S_{2 n}^{*}\right\|_{2} \leq 2\|X\|\left\|S_{2 n}\right\|_{2}
$$

and thus

$$
\left\|Q_{22}\right\|_{2} \rightarrow 0
$$

According to (4) and (5),

$$
\left\|Q_{41}+Q_{22}\right\|_{2} \leq\left\|Q_{41}\right\|_{2}+\left\|Q_{22}\right\|_{2} \lesssim\left\|R_{41}\right\|_{2},
$$


and consequently,

$$
\left\|Q P_{n}\right\|_{2} \lesssim\left\|R_{11}\right\|_{2}+\left\|R_{41}\right\|_{2}
$$

The proof will be finished after we establish that $\left\|R_{11}\right\|_{2}+\left\|R_{41}\right\|_{2}$ is bounded. Since $R \in \mathcal{C}_{2}(\mathcal{H}),\left\|R P_{n}\right\|_{2}^{2} \uparrow \operatorname{Tr}\left(R^{*} R\right)$, and thus $\left\|R P_{n}\right\|_{2}^{2}$ is bounded above by $\|R\|_{2}^{2}$.

The representation of $R$ relative to the decomposition of $\mathcal{H}$ into $P_{n} \mathcal{H} \oplus(I-$ $\left.P_{n}\right) \mathcal{H}$ is

$$
\left(\begin{array}{ll}
R_{11}+R_{23} & * \\
R_{41}+R_{33} & *
\end{array}\right)
$$

where $R_{23}=S_{2 n} X_{3 n}-X_{2 n} S_{3 n}$ and $R_{33}=S_{3 n} X_{1 n}-X_{4 n} S_{3 n}$, and therefore

$$
\left\|R P_{n}\right\|_{2}^{2}=\left\|R_{11}+R_{23}\right\|_{2}^{2}+\left\|R_{41}+R_{33}\right\|_{2}^{2} .
$$

On other hand,

$$
\left\|R_{11}\right\|_{2} \leq\left\|R_{11}+R_{23}\right\|_{2}+\left\|R_{23}\right\|_{2}
$$

and

$$
\left\|R_{23}\right\|_{2}^{2} \leq 2\|X\|^{2}\left(q_{2}^{2}(S)+q_{2}^{2}\left(S^{*}\right)\right) \rightarrow 0,
$$

and thus

$$
\left\|R_{11}\right\|_{2} \lesssim\left\|R_{11}+R_{23}\right\|_{2}
$$

Similarly

$$
\left\|R_{41}\right\|_{2} \leq\left\|R_{41}+R_{33}\right\|_{2}+\left\|R_{33}\right\|_{2}
$$

and

$$
\left\|R_{33}\right\|_{2} \rightarrow 0
$$

and therefore

$$
\left\|R_{41}\right\|_{2} \lesssim\left\|R_{41}+R_{33}\right\|_{2} .
$$

According to (9) and (10),

$$
\left\|R_{11}\right\|_{2}+\left\|R_{41}\right\|_{2} \lesssim\left\|R_{11}+R_{23}\right\|_{2}+\left\|R_{41}+R_{33}\right\|_{2},
$$

and thus

$$
\left\|R_{11}\right\|_{2}+\left\|R_{41}\right\|_{2} \lesssim\left\|R P_{n}\right\|_{2}
$$

Finally, according to (7) and (12),

$$
\left\|Q P_{n}\right\|_{2} \lesssim\left\|R P_{n}\right\|_{2}
$$

and thus

$$
\|Q\|_{2} \leq\|R\|_{2}
$$

which ends the proof.

Applying Proposition 2 to $S^{*}$, one obtains the following. 
Corollary 3. If $S \in L(\mathcal{H})$ is a normal operator and $X \in L(\mathcal{H})$ is such that $R:=S X-X S \in \mathcal{C}_{2}(\mathcal{H})$, then $Q:=S^{*} X-X S^{*} \in \mathcal{C}_{2}(\mathcal{H})$ and $\|Q\|_{2}=\|R\|_{2}$.

It is an elementary step to extend the above Corollary to two normal operators and to deduce the Hilbert-Schmidt norm equality obtained by G. Weiss.

A careful reviewing of the proof of Proposition 2 leads to the following.

Theorem 4. Let $S \in A N(\mathcal{H})$ satisfy $q_{2}(S)=q_{2}\left(S^{*}\right)=0$ and let $X \in L(\mathcal{H})$ such that $R:=S X-X S \in \mathcal{C}_{2}(\mathcal{H})$. Then $Q:=S^{*} X-X S^{*} \in \mathcal{C}_{2}(\mathcal{H})$ and $\|Q\|_{2} \leq\|R\|_{2}+c\|X\| \cdot\left\|D_{S}\right\|_{1}^{\frac{1}{2}}$, where $c>0$.

This implies, by a standard argument, the following.

Theorem 5. Let $S_{1}, S_{2} \in A N(\mathcal{H})$ satisfy $q_{2}\left(S_{1}\right)=q_{2}\left(S_{2}\right)=q_{2}\left(S_{1}^{*}\right)=$ $q_{2}\left(S_{2}^{*}\right)=0$ and let $X \in L(\mathcal{H})$ such that $R:=S_{1} X-X S_{2} \in \mathcal{C}_{2}(\mathcal{H})$. Then $Q:=S_{1}^{*} X-X S_{2}^{*} \in \mathcal{C}_{2}(\mathcal{H})$ and $\|Q\|_{2} \leq\|R\|_{2}+c\|X\| \cdot\left(\left\|D_{S_{1}}\right\|_{1}+\left\|D_{S_{2}}\right\|_{1}\right)^{\frac{1}{2}}$, where $c>0$.

Proof. Set $S=S_{1} \oplus S_{2}$ and observe that $S \in A N(\mathcal{H})$ and $q_{2}(S)=0$ since $q_{2}^{2}(S) \leq q_{2}^{2}\left(S_{1}\right)+q_{2}^{2}\left(S_{2}\right)$ and $\operatorname{Tr}\left(D_{S}\right)=\operatorname{Tr}\left(D_{S_{1}}\right)+\operatorname{Tr}\left(D_{S_{2}}\right)=0$, and thus $q_{2}\left(S^{*}\right)=0$, and apply Theorem 4 to get the desired conclusion.

The following is an example of a subnormal operator that is almost normal with modulus of $\mathcal{C}_{2}$-quasitriangularity and the trace of its self-commutator equal zero, such that the Hilbert-Schmidt norm of $R$ and $Q$ are not equal.

Example 6. Let $\mathcal{H}=l^{2}(\mathbb{N})$, and $S_{k}$ be the weighted (unilateral) shift with weights $\frac{1}{k}, \frac{1}{k+1}, \ldots$, let $S$ be a block-weighted shift defined on $l^{2}(\mathbb{N})^{(\infty)}$ in which the nonzero blocks are $S_{1}, S_{2}, \ldots$ Let $X \in L\left(l^{2}(\mathbb{N})^{(\infty)}\right)$ be the also a block-shift with all nonzero blocks equal to the identity, that is $X=S_{0} \otimes I$, where $S_{0}$ is the standard (unilateral) shift.

It is an entertaining exercise and is left to the reader to see that the operator $S$ satisfies the above properties and that $\|S X-X S\|_{2} \neq\left\|S^{*} X-X S^{*}\right\|_{2}$.

\section{References}

[1] T. Furuta, An extension of the Fuglede-Putnam theorem to subnormal operators using a Hilbert-Schmidt norm inequality, Proc. Amer. Math. Soc., 81 (1981), $240-242$.

[2] F. Kittaneh, On generalized Fuglede-Putnam theorems of Hilbert-Schmidt type, Proc. Amer.Math. Soc., 88 (1983), 293-298. 
[3] F. Kittaneh, A Fuglede-Putnam theorem for almost normal operators with finite modulus of $\mathcal{C}_{\in}$-quasitriangularity modulo the Hilbert-Schmidt class, Submitted.

[4] D. V. Voiculescu, Some extensions of quasitriangularity, Rev. Roumaine Math. Pures Appl., 18 (1973), 1303-1320.

[5] D. V. Voiculeascu, Some results on norm-ideal perturbation of Hilbert space operators, J. Operator Theory, 2 (1979), 3-37.

[6] D. V. Voiculescu, A note on quasitriangularity and trace-class selfcommutators, Acta Sci. Math. (Szeged), 42 (1980), 195-199.

[7] D. V. Voiculescu, Remarks on Hilbert-Schmidt perturbations of almost normal operators, Topics in Modern Operator Theory; Operator Theory: Advances and Applications-Birkhäuser, 2 (1981), 311-318.

[8] G. Weiss, Fuglede's commutativity theorem modulo the Hilbert-Schmidt class and generating functions for matrix operators. II, J. Operator Theory, 5 (1981), 3-16.

[9] G. Weiss, The Fuglede commutativity theorem modulo operator ideals, Proc. Amer.Math. Soc., 83 (1981), 113-118.

\section{Received: September 7, 2014}

